\title{
Gridless TEM Supports Promise Greater Experimental Throughput
}

\author{
P.S. Hollis* and F.R. Powell* \\ Luxel Corporation, P.O. Box 1879, Friday Harbor, WA 98250
}

We report on the results of a Phase I Small Business Innovation Research (SBIR) project to determine if a strong, beam stable, electron transparent TEM specimen support could be made without obstructing grid wires by employing nano-thin polyimide membranes. More than 650 sample TEM supports were fabricated during the project. 35 to $100 \mathrm{~nm}$ thick prototype supports were prepared on 100-mesh, $0.4 \times 2$ and $1 \times 2 \mathrm{~mm}$ slots, and $1.5 \mathrm{~mm}$ and $2.0 \mathrm{~mm}$ diameter circular open aperture blanks. These were examined in characterization studies and by volunteer evaluators in the course of their work in thin section imaging, cryoelectron microscopy, cellular tomography, automated electron microscopy, and immunocytochemistry.

The membranes proved strong and stable over unobstructed millimeter-scale apertures thus demonstrating the feasibility of gridless TEM supports. The studies showed that the polyimide grids possess good handling characteristics, both in adhering sections to the support as well as retaining sections during subsequent processing of sections. The supports performed well for both standard TEM imaging and more involved immunocytochemical studies. In a 7-month study employing the supports in thin section imaging studies of crab muscle tissue, only 3 out of more than 250 supports broke and these only due to mishandling. The polyimide films exhibited superior strength and beam stability compared to Formvar or Butvar, and anecdotal evidence suggests they have a longer useful shelf life. No image drift was reported. Circular open aperture supports, $2.0 \mathrm{~mm}$ in diameter, proved stable over long beam exposures needed to perform tilt series data collection for tomography without additional carbon coating.

The supports excelled in general imaging applications at low magnifications and at higher voltages. The higher contrast afforded by low beam voltage resolved a mottled background. This background was evident in some images of naked polyimide films at $80 \mathrm{keV}$, however, it did not interfere with section images or in tomographic reconstructions in most cases. This issue, along with film-to-grid adhesive selection, solvent compatibility; surface energy, and batch fabrication, remain topics for further research and development.

The stability under the beam and the large open viewing area suggests these supports may be an improved option for electron tomography and immunocytochemistry. In addition, the films are robust at cryogenic temperatures indicating they could be used to advantage in cryo electron microscopy of vitreous sections. Developing stable gridless supports for these applications would greatly improve the efficiency and throughput of TEM work by allowing the researcher to view the entire specimen; a significant improvement when looking at large structures, searching for details that occur infrequently in the tissue sample, or imaging serial sections of tissues to reconstruct the 3dimensional aspect of the structure of interest.

\section{References}

[1] This work was supported by NIH grant 1 R43 GM071098-1 (SBIR). We gratefully acknowledge grid evaluations and helpful discussions by Dr.'s B. Marsh and A. McDowall (U. Queensland); C. Meshul (V.A. Medical Center Portland); C. Potter (NRAMM); and S. Cain (Friday Harbor Labs). 
Table 1: Optimal exposure times in seconds for Polyimide of various thicknesses, Formvar, and uncoated grids. All Polyimide thicknesses (*) are in $\mathrm{nm}$.

\begin{tabular}{|c|c|c|c|c|c|c|c|c|}
\hline \multirow{5}{*}{ 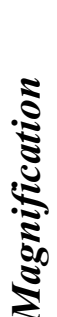 } & & \multicolumn{7}{|c|}{ Sample Type } \\
\hline & & No Film & 38.8* & $40.6^{*}$ & 50.0* & $55.7 *$ & $100.0 *$ & Formvar \\
\hline & $8,200 x$ & 1.5 & 2.0 & 2.1 & 2.2 & 2.0 & 2.4 & 2.1 \\
\hline & $60,000 x$ & 1.4 & 2.0 & 2.0 & 2.1 & 2.1 & 2.7 & 2.1 \\
\hline & $105,000 x$ & 1.5 & 2.0 & 2.2 & 2.5 & 2.5 & 3.1 & 2.1 \\
\hline
\end{tabular}
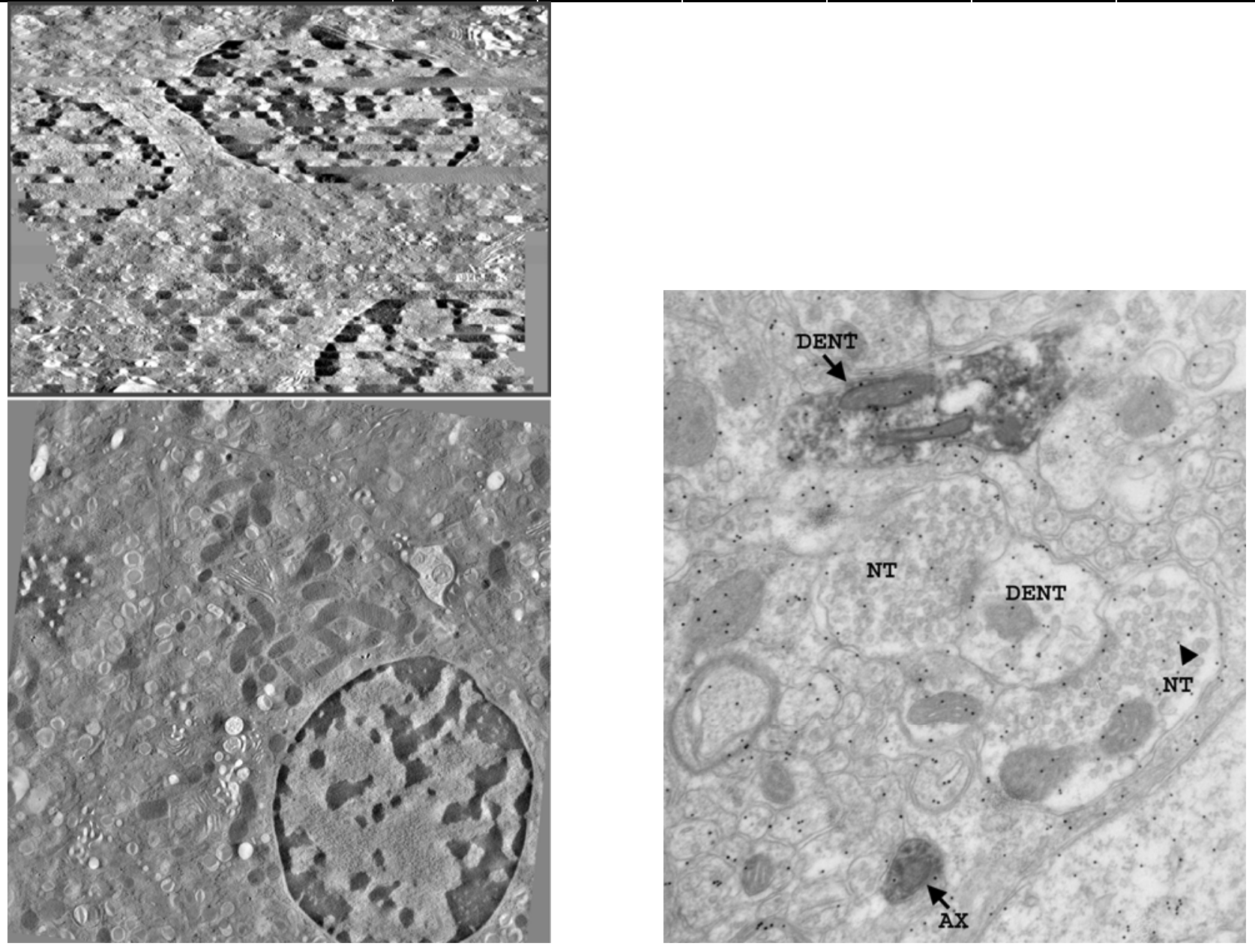

Figure 1 Gridless supports were evaluated in research applications that included cellular tomography (left) and immunocytochemistry (right). LEFT: Image panel from serial section reconstruction of insulin-secreting beta cells isolated from adult, female Balb/c mice. TOP: $z$-axis view of the 45 serial sections that together comprise the 3D cellular volume. BOTTOM: normal $\mathrm{x}-\mathrm{y}$ view. $2 \mathrm{~mm}$ gridless supports allowed tilt series data collection with no drift. The surface energy of the films complicated laying down the section ribbon, but the large open area mitigated this, as the ribbon did not need to be centered over a small slot. Image credit Brad Marsh, PhD, U. Queensland, Australia. RIGHT: Localization of two antibodies within mouse brain tissue. $1 \times 2 \mathrm{~mm}$ slot grids covered with $50 \mathrm{~nm}$ (on average) polyimide membranes allowed $80 \%$ more throughput (good images) than commonly used 200-mesh grids. Image credit Charles Meshul, PhD, Veterans Administration Medical Center, Portland, OR. 